\title{
In Planta Expression or Delivery of Potato Aphid Macrosiphum euphorbiae Effectors Me10 and Me23 Enhances Aphid Fecundity
}

\author{
Hagop S. Atamian, ${ }^{1,2}$ Ritu Chaudhary, ${ }^{1,2}$ Valeriano Dal Cin, ${ }^{2}$ Ergude Bao, ${ }^{3}$ Thomas Girke, ${ }^{1,4}$ and \\ Isgouhi Kaloshian ${ }^{1,2,5}$ \\ ${ }^{1}$ Graduate Program in Genetics, Genomics and Bioinformatics, ${ }^{2}$ Department of Nematology, ${ }^{3}$ Department of Computer \\ Science and Engineering, ${ }^{4}$ Department of Botany and Plant Sciences, and ${ }^{5}$ Center for Disease Vector Research, \\ University of California, Riverside, CA 92521, U.S.A.
}

Submitted 24 June 2012. Accepted 6 October 2012.

\begin{abstract}
The interactions between aphids and their host plants seem to be analogous to those of plant-microbial pathogens. Unlike microbial pathogen effectors, little is known about aphid effectors and their ability to interfere with host immunity. To date, only three functional aphid effectors have been reported. To identify potato aphid (Macrosiphum euphorbiae) effectors, we developed a salivary gland transcriptome using Illumina technology. We generated 85 million Illumina reads from salivary glands and assembled them into 646 contigs. $A b$ initio sequence analysis predicted secretion signal peptides in $24 \%$ of these sequences, suggesting that they might be secreted into the plant during aphid feeding. Eight of these candidate effectors with secretion signal peptides were functionally characterized using Agrobacterium tumefaciens-mediated transient overexpression in Nicotiana benthamiana. Two candidate effectors, Me10 and Me23, increased aphid fecundity, suggesting their ability to suppress $N$. benthamiana defenses. Five of these candidate effectors, including $\mathrm{Me10}$ and $\mathrm{Me23}$, were also analyzed in tomato by delivering them through the Pseudomonas syringae type three secretion system. In tomato, only Me10 increased aphid fecundity. This work identified two additional aphid effectors with ability to manipulate the host for their advantage.
\end{abstract}

Aphids (Hemiptera: Aphididae) are soft-bodied insects with piercing-sucking mouthparts that cause serious economic losses to cultivated crops. They damage plants directly, by depleting nutrients and altering plant development, and indirectly, by vectoring plant viruses and supporting growth of the sooty mold fungus (Blackman and Eastop 2000). Some aphid species are

\section{H. S. Atamian and R. Chaudhary contributed equally to this work.}

Nucleotide sequence data have been deposited in the DDBJ, EMBL, and GenBank databases under the following accession numbers: Me5 (JX134487), Me10 (JX134488), Me13 (JX134489), Me14 (JX134490), Me17 (JX134491), Me20 (JX134492), Me23 (JX134493), Me25 (JX134494).

Corresponding author: I. Kaloshian; E-mail: Isgouhi.kaloshian@ucr.edu

* The $\boldsymbol{e}$-Xtra logo stands for "electronic extra" and indicates that four supplementary tables are published online.

(C) 2013 The American Phytopathological Society globally distributed due to their polyphagous nature and ability to adapt to different environmental conditions (Margaritopoulos et al. 2009). The life cycle of aphids is somewhat complex, comprising both sexual and asexual (parthenogenetic) modes of reproduction, the latter giving rise to live progeny (Blackman and Eastop 2000). Sexual reproduction occurs only in the fall season when eggs are laid on perennial plants for overwintering. Being hemimetabolous insects, aphids have no morphologically distinct larval or pupal stages. During asexual reproduction, females lay nymphs which, after three successive molts become adults (Moran 1992; Van Emden and Harrington 2007).

The potato aphid (Macrosiphum euphorbiae) has a broad host range including plants in family Solanaceae, transmits a number of plant viruses, and represents an aphid species of worldwide significance (Chan et al. 1991; Moeller 1973; Radcliffe and Ragsdale 2002). Its abundance and propensity to develop alatae makes this aphid species very important in viral epidemiology (Cerato et al. 1994; Singh and Boiteau 1986). In tomato (Solanum lycopersicum), resistance to potato aphids is mediated by the Mi-1 gene, which encodes a coiled-coil nucleotide-binding leucine-rich protein and requires the Somatic Embryogenesis Receptor Kinase 1 (SlSERK1) (Mantelin et al. 2011; Rossi et al. 1998). The aphid effector recognized directly or indirectly by $\mathrm{Mi}-1$ remains elusive.

Aphids are phloem feeders that use a pair of slender stylets to mostly move between cells until they reach the sieve element, where they feed for a prolonged period of time. Although the stylet's path is mainly intercellular, stylets do also puncture cells and cause cell-wall disturbance and damage to the plasma membranes of mesophyll and parenchyma cells (Moran et al. 2002; Pollard 1973; Tjallingii and Hogen Esch 1993). Unlike chewing insects that cause extensive tissue damage, this specialized aphid feeding behavior and interaction with its host, avoiding extensive mechanical tissue damage, is analogous to plant-biotrophic pathogen interactions in which the pathogen is sustained in a localized area and is dependent on living host plant cells. Consistent with these observations, induction of plant genes associated with pathogen-induced response pathways have been reported as a result of aphid feeding (De Vos et al. 2005; Kaloshian and Walling 2005; Martinez de Ilarduya et al. 2003; Thompson and Goggin 2006).

Aphids release two types of saliva during feeding, soluble saliva and gelling saliva. The soluble saliva is in liquid form and is delivered along the penetration path and in the sieve element, whereas the gelling saliva forms a proteinaceous sheath around the stylets as soon as it exits the stylet tip (Miles 1999; 
Tjallingii 2006). It has been hypothesized that constituents of aphid salivary secretions play crucial roles in modulating plant responses. Aphid saliva consists of a suite of bio-reactive compounds, some of which may serve as cues to elicit plant defenses, while others are expected to function in suppressing or circumventing plant defenses (Harris et al. 2003; Hogenhout and Bos 2011; Miles 1999).

It is speculated that aphid-host interactions shadow the commonly accepted zig-zag model of plant-microbial pathogen defense evolution described by Jones and Dangl (2006). In pathosystems, conserved sets of molecular signatures called pathogen- or microbe-associated molecular patterns (PAMPs or MAMPs) or general elicitors are recognized by pattern recognition receptors (PRR) present at the host cell surface. PRR-mediated non-self recognition activates pattern-triggered immunity (PTI) in the host plant. PTI is associated with activation of downstream signaling pathways, including mitogenactivated protein kinases and WRKY transcription factors, induction of defense responses, including production of reactive oxygen species, accumulation of pathogen-related (PR) proteins, and callose deposition that collectively restrict microbial growth (Segonzac and Zipfel 2011). Pathogens can secrete small molecules or proteins known as effectors (Abramovitch et al. 2006; Birch et al. 2006; Davis et al. 2008; Kamoun 2006; van der Hoorn 2008) to counteract PTI and effectively parasitize and colonize the host plant. Extensive research using various approaches has led to the identification of hundreds of effectors secreted by bacterial, fungal, and oomycete plant pathogens that were essential for understanding pathogenesis.

Unlike PAMPs and pathogen effectors, which have been extensively studied during the past decade, not much is known about herbivore-associated molecular patterns or herbivore effectors (Hogenhout and Bos 2011; Miles 1999; Tjallingii 2006). One or more proteinaceous elicitors with a size between 3 and $10 \mathrm{kDa}$ in the saliva of green peach aphid Myzus persicae have been shown to induce defense responses in Arabidopsis (De Vos and Jander 2009). Similarly, expressing the Myzus persicae proteins $M p 10$ and Mp42 in Nicotiana benthamiana resulted in a decrease in aphid fecundity (Bos et al. 2010). Moreover, induction of plant defense genes by feeding of piercing-sucking herbivores, including aphids, has been extensively demonstrated (Kempema et al. 2007; Martinez de Ilarduya et al. 2003; Rodriguez-Saona et al. 2010; Thompson and Goggin 2006). In contrast, little is known about how the salivary proteins of piercing-sucking herbivores may interfere with the plant immune system or manipulate host metabolites for their advantage. Aphids alter host primary metabolism and improve nutrient composition of the phloem sap to enhance their growth (Giordanengo et al 2010; Wilson et al 2011). However, it is not clear how aphids are able to cause these changes or the one or more salivary secretions responsible for these changes. A few aphid salivary proteins have been implicated in enhancing aphid performance. The salivary protein $M p C 002$ has been shown to enhance Myzus persicae fecundity or nymph production on $N$. benthamiana (Bos et al. 2010). In vitro analysis of the effect of the vetch aphid Megoura viciae saliva on forisomes provided direct evidence that aphid saliva has the ability to counteract plant defenses and prevent sieve tube plugging, providing aphids with access to a continuous flow of phloem sap (Will et al. 2007). Moreover, some piercing-sucking insects have been shown to suppress the expression of plant defense genes and manipulate defense signaling pathways to their advantage (Zarate et al. 2007; Zhang et al. 2011). Taken together, the ultimate outcome of interactions of piercing-sucking herbivores with their host plant is likely to depend on the salivary secretion and effectors produced by the herbivore and the ability of the plant to perceive these effectors and respond appro- priately as well as the ability of the salivary secretions to alter host metabolism.

A large number of pathogen effectors have been identified using homology-based searches, while others were likely missed as their sequences are unique. In aphids, candidate effector molecules have been identified using three approaches involving i) sequencing the aphid salivary gland transcriptome or ii) the salivary gland proteome, followed by prediction of the protein secretion signal, or iii) by direct sequencing the aphid salivary proteome (Bos et al. 2010; Carolan et al. 2009, 2011; Harmel et al. 2008). In this study, we sequenced the salivary gland transcriptome of $M$. euphorbiae, using the Illumina sequencing platform, and assembled the reads into 646 contigs. Data mining of the assembled contigs identified 159 predicted $M$. euphorbiaesecreted proteins. Eight candidate effectors with secretion-signal peptides identified in the salivary transcriptome were functionally characterized, using Agrobacterium tumefaciens-mediated transient overexpression in $N$. benthamiana or delivered by the Pseudomonas syringae type three secretion system (T3SS) into tomato plants. Using these assays, we identified two effectors, $M e 10$ and $M e 23$, that enhanced aphid fecundity.

\section{RESULTS AND DISCUSSION}

\section{Transcriptome sequencing, assembly, and annotation.}

To analyze the $M$. euphorbiae salivary gland transcriptome, an RNA-Seq library was prepared from 200 dissected salivary glands of adult aphids. To determine the quality of the library, an aliquot was cloned and 10 clones were subjected to Sanger sequencing. TBLASTX analysis of the clones against the nonredundant (nr) database at the National Center for Biotechnology Information (NCBI) identified unique sequences in each clone, suggesting that the library was not biased for certain transcripts.

A total of 85 million reads were generated from this RNASeq library, of which 4 million were unique. Initially, the generated reads were assembled using the Velvet/Oases assembler (Schulz et al. 2012). Further improvement of the assembly was achieved by reducing the redundancy in the data set with the SEED program, novel clustering algorithm for next-generation sequencing data, which resulted in longer contigs (Bao et al. 2011). In total, 646 contigs were generated, with $62 \%$ of the assembled contigs longer than $500 \mathrm{bp}$.

To determine how many of the $M$. euphorbiae salivary gland contigs have putative orthologs in the closely related species Acyrthosiphon pisum, whose genome has been sequenced, reciprocal TBLASTX analysis was performed. A total of $551 \mathrm{M}$. euphorbiae contigs were identified with sequences orthologous to $460 \mathrm{~A}$. pisum transcripts (Supplementary Table S1). Some of the $M$. euphorbiae contigs matched to non-overlapping regions of the same $A$. pisum transcript, explaining the larger number of $M$. euphorbiae orthologous contigs. Consequently, these contigs were annotated according to version 2 of the $A$. pisum annotation (International Aphid Genomics Consortium 2010). Out of the $460 \mathrm{~A}$. pisum transcripts identified in this study, 155 were also identified in the A. pisum salivary glands by Carolan and associates (2011). Putative orthologs for an additional 41 contigs were identified by performing reciprocal BLASTX analysis against the Uniprot database and were annotated accordingly. Although these $M$. euphorbiae contigs did not have orthologs in A. pisum, we cannot exclude the possibility that they may be present in other aphid species. Orthologs could not be identified for $54 \mathrm{M}$. euphorbiae contigs. They could be the result of inaccurate assembly of the Illumina reads, although it is possible that some of these sequences are correctly assembled and consequently unique to M. euphorbiae. 
To determine the putative functions of the $M$. euphorbiae contigs, we used the database containing gene ontology (GO) assignments of all the publicly available A. pisum expressed sequence tags (EST). The functional classification of the contigs based on GO terms showed enrichment for the classes "translation", "metabolic process", and "transport" in the GO category "biological process" (Fig. 1A). Aphids have a pair of salivary glands, each consisting of a principal gland and two accessory glands (Ponsen 1972; Weidemann 1968). Besides the expected cell maintenance processes, the cells of the salivary glands undergo cycles of secretory activities (Miles 1999). Thus, transcripts grouped under the "translation" category are likely to serve both functions. To fulfill their respective roles, those proteins destined to be delivered in the saliva and potentially having roles in the interactions with the plant host are expected to have secretion signals allowing them to cross cell membranes into the salivary canal. Consequently, it is not surprising that transcripts predicted to have a transport function were enriched in this organ. On the other hand, the GO terms in the "molecular function" category showed more distributed and diverse enrichments of various molecular activity categories (Fig. 1B).

Identification of sequences with secretion signal peptides.

The amino acid sequences of the putative full-length $A$. pisum orthologs of the identified $M$. euphorbiae contigs were analyzed with the SignalP 4.0 (Petersen et al. 2011) and TargetP (Emanuelsson et al. 2000) prediction software programs trained to identify signal peptides. Of the 460 examined sequences, 125 and 159 were predicted to have putative signal peptides predicted by SignalP 4.0 (hidden Markov model scores higher than 0.45) and TargetP (predefined set of cutoffs that yields specificity $>0.95$ on the TargetP test sets), respectively. TargetP predicted a signal peptide in 121 sequences predicted by SignalP. Moreover, it predicted an additional 38 sequences that were not predicted by SignalP. In the A. pisum salivary gland transcriptome, $30 \%$ (324 of 1,074) of the transcripts were predicted to have signal peptides using SignalP 3.0 (Carolan et al. 2011). Using the version 4.0 of the same program we predicted $27 \%$ (125 of 460) of the salivary gland transcripts to have signal peptide. Around $42 \%$ of the sequences with predicted signal peptides in this study were also identified by Carolan and associates (2011). This low overlap of sequences with signal peptides between the two studies suggests that the salivary gland sequences from both species are incomplete.

\section{Selection of clones for functional analysis and their tissue-specific expression.}

An efficient way to investigate the roles of aphid candidate effectors in planta is to transiently overexpress them in N. benthamiana, using Agrobacterium tumefaciens, and assay with a population of aphids that are adapted to plants with nicotine, such as Myzus persicae. A similar approach, using N. benthamiana leaf disks, was successfully used to evaluate Myzus persicae effectors (Bos et al. 2010). In order to use this assay system,

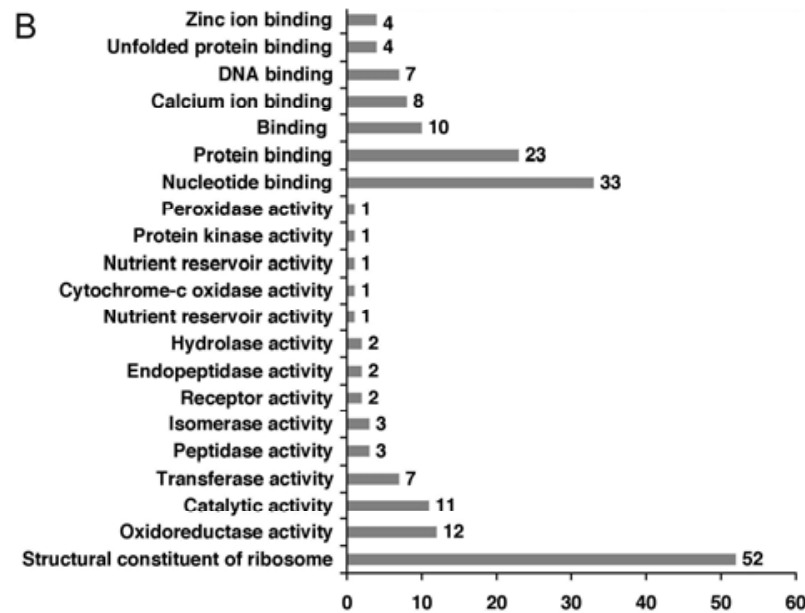

Fig. 1. Classification of the Macrosiphum euphorbiae contigs using the Acyrthosiuphon pisum Gene Ontology terms. A, The contigs were annotated according to the biological process that they are predicted or known to be part of or $\mathbf{B}$, the known or predicted molecular function.

Table 1. Macrosiphum euphorbiae selected genes with predicted secretion peptide and their Acyrthosiphum pisum and Myzus persicae putative orthologs

\begin{tabular}{|c|c|c|c|c|c|c|c|c|}
\hline \multirow[b]{2}{*}{ Gene } & \multirow[b]{2}{*}{ ID $^{\mathbf{a}}$} & \multirow[b]{2}{*}{ Size $^{\mathbf{b}}$} & \multicolumn{2}{|c|}{ Ortholog and E-value } & \multirow[b]{2}{*}{ Conserved domain } & \multirow[b]{2}{*}{ Annotation } & \multicolumn{2}{|c|}{ Phenotype $^{\mathrm{c}}$} \\
\hline & & & A. pisum & Myzus persicae & & & N. $b$. & Tomato \\
\hline$M e 5$ & Me_SG098 & 1,713 & ACYPI002298 $\left(10^{-193}\right)$ & $M p$ Contig8632 $\left(10^{-45}\right)$ & $\begin{array}{l}\text { Bacterial alpha-L- } \\
\text { rhamnosidase }\end{array}$ & Trehalase & No & NA \\
\hline$M e 10$ & Me_SG525 & 387 & ACYPI008224 $\left(10^{-69}\right)$ & $M p$ Contig6453 $\left(10^{-49}\right)$ & No conserved domain & Uncharacterized & Yes & Yes \\
\hline$M e 13$ & $M e \_S G 442$ & 324 & ACYPI56566 $\left(10^{-63}\right)$ & $M p$ Contig11439 $\left(10^{-47}\right)$ & $\begin{array}{l}\text { Cytochrome } \mathrm{P} 450 \text {, } \\
\text { Spumavirus gag protein }\end{array}$ & Uncharacterized & No & NA \\
\hline$M e 14$ & Me_SG355 & 954 & ACYPI21663 $\left(10^{-178}\right)$ & MpContig3168 $\left(10^{-129}\right)$ & Lipase superfamily & Lipase & No & No \\
\hline Me17 & $M e \_S G 427$ & 1,014 & ACYPI53825 $\left(10^{-152}\right)$ & $M p$ Contig11230 $\left(10^{-103}\right)$ & $\begin{array}{l}\text { Tat binding protein 1- } \\
\text { interacting protein }\end{array}$ & Hypothetical & No & No \\
\hline$M e 20$ & Me_SG497 & 1,131 & ACYPI21412 $\left(10^{-166}\right)$ & $M p$ Contig $1043\left(10^{-57}\right)$ & No conserved domain & Hypothetical & No & NA \\
\hline$M e 23$ & Me_SG130 & 636 & ACYPI002439 $\left(10^{-122}\right)$ & $M p$ Contig1685 $\left(10^{-93}\right)$ & Glutathione peroxidase & Glutathione peroxidase & Yes & No \\
\hline$M e 25$ & $M e \_S G 229$ & 918 & ACYPI006300 $\left(10^{-177}\right)$ & $M p$ Contig2442 $\left(10^{-81}\right)$ & $\begin{array}{l}\text { Carbonic anhydrase alpha } \\
\text { (vertebrate-like) }\end{array}$ & $\begin{array}{l}\text { Carbonic anhydrase } \\
\text { 2-like }\end{array}$ & No & No \\
\hline
\end{tabular}

\footnotetext{
${ }^{a} M$. euphorbiae expressed sequence tag indentification number.

${ }^{\mathrm{b}}$ Given in bp, excluding the signal peptide.

${ }^{\mathrm{c}}$ N. $b .=$ Nicotiana benthamiana $; \mathrm{NA}=$ not tested.
} 
we chose $M$. euphorbiae effectors with secretion signals and putative orthologs in Myzus persicae. To identify the Myzus persicae orthologs of our set of salivary gland-expressed $M$. euphorbiae contigs, we reassembled the publicly available Myzus persicae EST and used them in reciprocal TBLASTX analysis with the $M$. euphorbiae salivary gland transcripts. We chose eight $M$. euphorbiae salivary gland contigs with putative orthologs in Myzus persicae, four with annotations, and four encoding yet-uncharacterized proteins (Table 1). We cloned the $M$. euphorbiae open reading frames (ORF) encoding the mature proteins corresponding to these sequences, excluding the signal peptide. These eight ORF (Me5, Me10, Me13, $\mathrm{Me} 14, \mathrm{Me}$ 17, $\mathrm{Me} 20, \mathrm{Me} 23$, and $\mathrm{Me25}$ ) have 72 to $85 \%$ amino acid-sequence identity to the corresponding Myzus persicae EST contig (Table 1). The relationship among the Myzus persicae and $A$. pisum orthologs of these eight $M$. euphorbiae clones was also demonstrated by reciprocal TBLASTX comparisons.

Four proteins were chosen for further study based on possible effector functions suggested by their annotation. Based on A. pisum annotation, Me5 encodes a trehalase. Trehalose accumulation is associated with Arabidopsis defense against aphids (Singh et al. 2011). Therefore, trehalase secreted by the aphid may hydrolyze trehalose and counteract host defenses. Me14 encodes a lipase; members of this superfamily of proteins have diverse roles including defense against oxidative stress (Horne et al. 2009). Me23 encodes a glutathione peroxidase (GPX) with a potentially protective role against oxidative burst (Lamb and Dixon 1997), while Me25 encodes a carbonic anhydrase 2, which may function in catalysis of aldehydes induced during aphid feeding (Gosset et al. 2009). Of the four uncharacterized proteins, conserved domain search identified known domains for two, Me13 and Me17 (Table 1).

To confirm that the eight genes were expressed in the salivary glands, we evaluated the accumulation of their transcripts in dissected head and glands or gut tissues of $M$. euphorbiae (Fig. 2). Seven genes were expressed in the head and glands

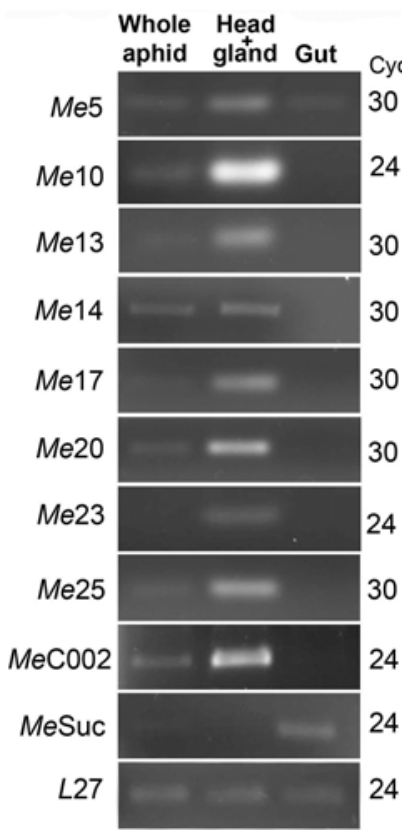

Fig. 2. Tissue-specific expression analysis of the eight Macrosiphum euphorbiae candidate effectors. RNA isolated from whole aphids or dissected aphid heads and salivary glands or guts was used in reverse transcription and semiquantitative polymerase chain reaction (PCR) with gene-specific primers. PCR cycles are indicated to the right of the panel. Expression of $\mathrm{MeCOO2}$ and $\mathrm{MeSuc}$ were used as controls for salivary glands and gut, respectively. Ribosomal gene $L 27$ was used as an internal control for cDNA. and not in the gut suggesting that their corresponding proteins are produced in the salivary glands, while $\mathrm{Me} 5$ transcripts were detected in all the tissues tested suggesting either tissueunspecific expression of this gene or expression of its paralogs in different tissues. As seven of the genes tested exhibited tissue-specific expression, it was unlikely that our tissue-specific cDNAs were contaminated. Orthologous transcripts of five of these genes, $M e 10, M e 13, M e 17, M e 20$, and $M e 23$, were also identified in $A$. pisum salivary gland transcriptome, while peptides corresponding to Me5, Me10, and Me23 orthologs were identified in the A. pisum salivary gland proteome (Carolan et al. 2011). The presence of transcripts to most of our selected salivary gland genes in both A. pisum and Myzus persicae, aphid species with narrow and broad host ranges, respectively, suggests a general rather than specialized roles for these genes.

\section{Evaluating the role of candidate $M$. euphorbiae effectors in aphid defense in $N$. benthamiana.}

To investigate the roles of $M$. euphorbiae candidate effectors in planta, we transiently expressed the selected $M$. euphorbiae proteins in $N$. benthamiana and assayed with a population of Myzus persicae adapted to feeding on tobacco (Kim and Jander 2007). For the transient expression assays, a large area of a $N$. benthamiana leaf was agroinfiltrated with the recombinant binary pEarleyGate100 vector expressing a candidate $M$. euphorbiae protein. Expression of green fluorescent protein (GFP) was used as control to monitor expression and aphid fecundity. At $24 \mathrm{~h}$ after infiltration, four Myzus persicae adults were caged on each infiltrated leaf exposing the infiltrated area to aphids. The following day, the adults and newly born nymphs were removed, leaving four first-instar nymphs. These remaining aphids were moved to a fresh plant with recently agroinfiltrated leaves on day 6 , day 9 , and day 12 . This schedule was based on efficient GFP expression. These nymphs became adults on day 8 , and their fecundity was evaluated on days 9,12 , and 15 . The average nymph production per aphid was calculated and normalized to those on the GFP control. Two candidate effectors, $M e 10(P=$ $0.004)$ and $M e 23(P=0.01)$, significantly increased Myzus persicae fecundity compared with that in the GFP control (Fig. 3). There was no significant difference in aphid fecundity on the plants expressing the remaining six effectors (Fig. 3). Although the increase in aphid fecundity on plants expressing Me10 or $M e 23$ was modest, this increase in aphid performance was consistent in three independent experiments. This suggests that $\mathrm{Me1O}$ and $\mathrm{Me} 23$ altered $\mathrm{N}$. benthamiana responses for the aphid's advantage. Contributions of a single effector in manipulating host responses could be minor and this effect could be amplified when combined with additional components of the cocktail of effectors secreted by the aphid. Therefore, simultaneous expression of a combination of effectors, with no effect individually, may also result in enhancement of aphid performance.

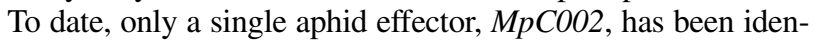
tified to increase aphid fecundity (Bos et al. 2010). Although it remains unknown how $\mathrm{MpCOO2}$ alters $\mathrm{N}$. benthamiana responses to Myzus persicae, the A. pisum ortholog ApCOO2 has been implicated in aphid orientation and feeding (Mutti et al. 2008). ApCOO2 is also secreted inside the plant host (Mutti et al. 2008). To date, nine and 17 salivary proteins have been identified from A. pisum and Myzus persicae, respectively (Carolan et al. 2009; Harmel et al. 2008). Interestingly, one of the $A$. pisum salivary proteins is the ortholog of Me10, the effector with an unknown function. The second $M$. euphorbiae effector gene we identified to increase aphid fecundity, $M e 23$, is predicted to encode a GPX and could be involved in reducing $\mathrm{H}_{2} \mathrm{O}_{2}$ and to function as an antioxidant to enhance aphid virulence and reduce the effect of the oxidative burst triggered by aphid feeding (Martinez de Ilarduya et al. 2003). 
Evaluating the role of candidate $M$. euphorbiae effectors in aphid defense in tomato.

To feed, aphids probe the plant host tissue with a pair of stylets and secret both watery and gelling saliva in this process. During probing and stylet penetration, cells are punctured and saliva is delivered inside the host cytoplasm. Therefore, aphid feeding can be compared with gram-negative plant pathogenic bacteria that possess a T3SS to invade and colonize the host cell by injecting virulence effectors (Buttner and He 2009; Cornelis and Van Gijsegem 2000). Therefore, we took the advantage of an existing bacterial system to deliver candidate $M$. euphorbiae effectors into the tomato cells. The T3SS of the bacterial pathogen $P$. syringae pv. tomato have been successfully utilized to deliver oomycyte Hyaloperonospora parasitica effectors into Arabidopsis cells (Rentel et al. 2008; Sohn et al. 2007). We characterized the function of five of the eight candidate effectors tested in $N$. benthamiana by delivering them through the $P$. syringae pv. tomato $\mathrm{T} 3 \mathrm{SS}$ into the tomato cell cytoplasm and assaying aphid performance on the plants. To deliver aphid effectors through the T3SS, we used the pVSP_PsSPdes expression vector, which has the promoter and secretion translocation signal of the $P$. syringae pv. tomato effector AvrRpm1 (Guttman and Greenberg 2001; Rentel et al. 2008). We introduced this vector in a less virulent strain of $P$. syringae pv. tomato DC3000, DC3000 $\Delta a v r P t o / \Delta a v r P t o B$ (Nguyen et al. 2010), lacking the two strong virulent effectors avrPto and $a v r P t o B$, to minimize the interference of bacterial effectors to better evaluate subtle differences in aphid performance. The roles of the five aphid effectors were evaluated in tomato by assessing their effect on M. euphorbiae fecundity. The Myzus persicae colony used in this study is not adapted to tomato and, therefore, was not used in the tomato assays. To perform the $M$. euphorbiae fecundity assays, 4- to 5-week-old tomato plants were vacuum-infiltrated with the recombinant $P$. syringae pv. tomato expressing the aphid constructs or $\beta$-glucuronidase (GUS) as control. Each plant was infested with nine 1-day-old, age-synchronized, adult aphids $24 \mathrm{~h}$ postinfiltration. Nymph production was evaluated

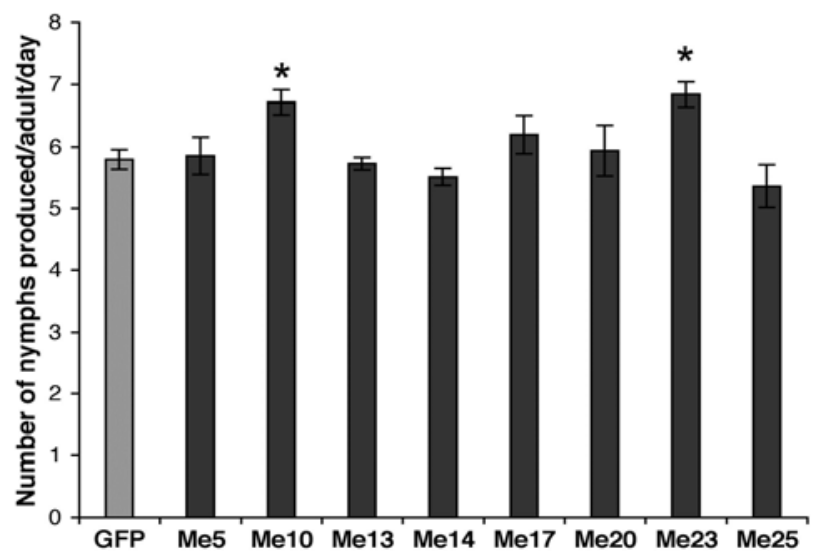

Fig. 3. Myzus persicae performance on Nicotiana benthamiana plants expressing Macrosiphum euphorbiae candidate effectors. $M$. euphorbiae candidate effectors were transiently overexpressed in $N$. benthamiana using Agrobacterium tumefaciens. One day after agroinfiltration, each leaf sample was caged with four adult Myzus persicae. The following day, adults were removed, leaving four first-instar nymphs. The four nymphs were moved to a freshly agroinfiltrated leaf expressing the candidate effector on days 6, 9, and 12. Nymph production was evaluated up to day 15 . Green fluorescent protein (GFP) was used as expression and aphid assay control. Graphs show the average number of nymphs produced per adult from one experiment. Two leaves per plant and four plants per construct were used $(n=8)$. Data from one experiment are presented, with error bars indicating the standard error. Asterisks indicate statistical significance compared with the GFP control (Me10 $P=0.004$; Me23 $P=0.01$ ). This experiment was performed three times with essentially identical results. for the next 5 days. The average nymph production per aphid per day was calculated and compared with the GUS control. Of the five candidate effectors tested, only $\mathrm{Me} 10$ significantly increased $(P=0.021)$ aphid fecundity on tomato (Fig. 4). Since Mel0 encodes a protein of 128 amino acids, it is likely that it was delivered into the host cell through the $P$. syringae pv. tomato T3SS and that the delivery of Me10 into the host cells resulted in the enhanced aphid fecundity phenotype. Thus, Me10 is able to manipulate tomato plant for the aphid's advantage, as it did in $N$. benthamiana. None of the remaining effectors affected aphid fecundity (Fig. 4). We did not find statistically significant differences in aphid performance in tomato plants infected with $P$. syringae pv. tomato expressing Me23 compared with the GUS control, suggesting that $M e 23$ is not able to alter tomato responses to detectable levels. It is possible that the different mode of effector delivery, the altered aphid assay used for tomato compared with $N$. benthamiana, or the shorter time exposure of aphids to plant expressing the effector did not allow detection of subtle differences in plant responses. Alternatively, $N$. benthamiana may be less tolerant than tomato to aphid infestation and, therefore, more suitable to detect the weak effects of $M e 23$ (Goodin et al. 2008). Since $\mathrm{Mel} 10$ is uncharacterized and has no known functionally conserved domains, it is difficult to speculate how it manipulates plant responses. Future experiments should elucidate this role. Nevertheless, this experiment showed that $P$. syringae pv. tomato T3SS can be used for delivery of aphid effectors in planta to evaluate aphid performance. This approach will allow the evaluation of aphid effectors without the need to express them by developing stable transgenic plants in hosts in which Agrobacterium-mediated transient expression does not work consistently or is not feasible to perform.

\section{$M e 10$ and $M e 23$ and their putative $A$. pisum and Myzus persicae orthologs.}

Two genes of the A. pisum genome (ACYPI002439 and ACYPI38240) are annotated as glutathione peroxidases. The amino acid sequence identity between $M e 23$ and the $A$. pisum

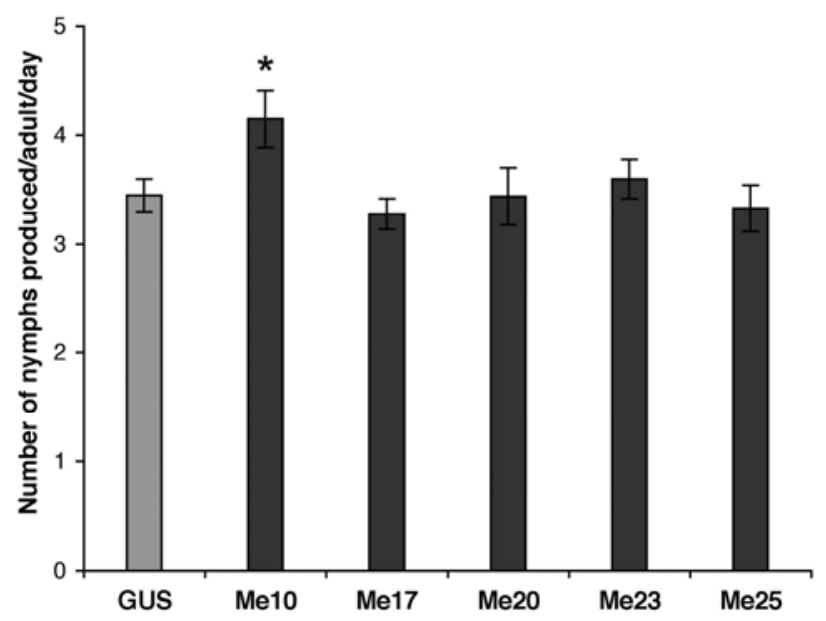

Fig. 4. Macrosiphum euphorbiae performance on tomato plants expressing M. euphorbiae candidate effectors. Five-week-old tomato plants were vacuum-infiltrated with recombinant Pseudomonas syringae pv. tomato

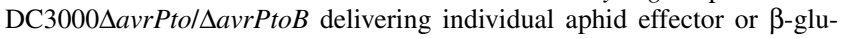
curonidase (GUS) used as control. Five plants per treatment were vacuuminfiltrated with recombinant $P$. syringae pv. tomato at $1 \times 10^{3} \mathrm{CFU} / \mathrm{ml}$, and at $24 \mathrm{~h}$ postinfiltration, each plant was infested with nine age-synchronized 1-day-old adult potato aphids. Aphid progeny were evaluated for 5 days. Graphs show the average number of nymphs produced per adult aphid $(n=$ 5). Data from one experiment are presented with error bars indicating the standard error. Asterisk indicates statistical significance compared with the GUS $(P=0.021)$. This experiment was performed three times with essentially identical results. 
proteins ACYPI002439 and ACYPI38240 is 87 and 42\%, respectively, while the amino acid sequence identity between Me23 and a putative Myzus persicae ortholog is 70\% (Table $1)$. It is not clear how many $M e 23$ orthologs are present in the Myzus persicae genome, as the genome of this aphid has not been sequenced. The alignment of the amino acid sequences from the three aphid species shows blocks of conserved regions (Fig. 5). Near the $\mathrm{N}$ terminus within one of these conserved regions, a deletion of eight amino acids is present in the Myzus persicae protein. The presence of such an insertion or deletion might alter the protein's function.

Me10 encodes an uncharacterized protein. BLAST analyses indicated that MelO is a single-copy gene in A. pisum (Table 1). The amino acid sequence of $M e 10$ has 76 and $88 \%$ sequence identity to Myzus persicae and A. pisum orthologs, respectively. Although these three aphids belong to the same tribe, Macrosiphini, within the aphid subfamily Aphidinae, it is not surprising that $M$. euphorbiae sequences are more similar to A. pisum, since $M$. euphorbiae is phylogenetically more closely related to A. pisum than Myzus persicae (Von Dohlen et al. 2006).

\section{MATERIALS AND METHODS}

\section{Plant material and aphid colonies.}

Tomato (Solanum lycopersicum) cultivars UC82B and Moneymaker, Nicotiana benthamiana, and tobacco (Nicotiana tabacum) NC-95 were used. Seedlings were transplanted into California mix II or sand. The plants were maintained in growth rooms at $24^{\circ} \mathrm{C}$ with a 16 -h-light and 8 -h-dark photoperiod and $200 \mu \mathrm{mol} \mathrm{m} \mathrm{m}^{-2} \mathrm{~s}^{-1}$ light intensity and were fertilized weekly with MiracleGro (18-18-21; Stern's MiracleGro Products, Port Washington, NY, U.S.A.).

Colonies of the parthenogenetic potato aphid (Macrosiphum euphorbiae) and green peach aphid (Myzus persicae) were reared on tomato cultivar UC82B and tobacco NC-95 plants, respectively. The colonies were maintained in insect cages in a pesticide-free greenhouse at 22 to $26^{\circ} \mathrm{C}$ supplemented with light for a 16-h day length. Age-synchronized, 1-day-old, adult M. euphorbiae aphids were produced as described by Bhattarai and associates (2007).

\section{RNA extraction and cDNA synthesis.}

RNA was isolated from 200 dissected $M$. euphorbiae salivary glands and was used for RNA-Seq library preparation as described previously (Atamian and Kaloshian 2012). Similarly, RNA was isolated from whole aphids and 20 dissected heads and salivary glands or guts. cDNA was synthesized from $100 \mathrm{ng}$ of DNase-treated whole body, head and salivary glands, or gut RNA using Superscript III (Invitrogen, Carlsbad, CA, U.S.A.) reverse transcriptase enzyme and oligo-dT primers according to the manufacturer's recommendations.

Library construction, sequencing, and de novo assembly.

A detailed procedure of RNA-Seq library preparation from salivary gland tissues has been described previously (Atamian and Kaloshian 2012). One lane of single-end 75-nucleotide long sequencing was performed with an Illumina Genome Analyzer II instrument at the Institute for Integrative Genome Biology, University of California Riverside. Data from the Illumina sequencing run was processed using the Illumina standard pipeline version 1.4. The sequence data generated in this study have been deposited in NCBI's Short Read Archive, accession number SRR547988. Redundancies in the data set were removed with the SEED NGS clustering tool (Bao et al. 2011). The remaining sequences were assembled with the Velvet/Oases assembler (Bao et al. 2011; Schulz et al. 2012). This Transcriptome Shotgun Assembly project has been deposited in the DDBJ, EMBL, and GenBank databases under the accession GAAF00000000 or presented in Supplementary Table S2. The version described in this paper is the first version, GAAF01000000.

\section{Annotation and secretion signal prediction.}

Reciprocal TBLASTX analyses were performed between $M$. euphorbiae and Acyrthosiphon pisum sequences to identify the putative orthologs of the $M$. euphorbiae sequences generated in this study. These putative orthologs were annotated based on the latest A. pisum annotation (aphidbase_2.1_peptides). The $M$. euphorbiae sequences with no putative orthologs in $A$. pisum were annotated by performing reciprocal TBLASTX analysis against the NCBI nucleotide (nt/nr) database. The annotated sequences were assigned to different $\mathrm{GO}$ categories based on available GO analysis. The full-length putative A. pisum ortho$\log$ s of the $M$. euphorbiae sequences were subjected to de novo signal peptide prediction analysis using SignalP 4.0 and TargetP 1.1 programs.

\section{Cloning M. euphorbiae salivary gland EST sequences.}

Eight $M$. euphorbiae salivary gland EST sequences with predicted secretion signal in their putative A. pisum orthologs were cloned. Gene-specific primers were designed, excluding the secretion signal peptide at the $5^{\prime}$ end, based on the A. pisum full-length sequences, and a start codon was added (Supplementary Table S3). The polymerase chain reaction (PCR)amplified products, obtained using Phusion high-fidelity polymerase (New England BioLabs, Beverly, MA, U.S.A.) were cloned into the pDONR207 (Invitrogen), were recombined into the binary vector pEarleyGate100 (Earley et al. 2006), and were sequenced using Sanger sequencing. The clones were trans-

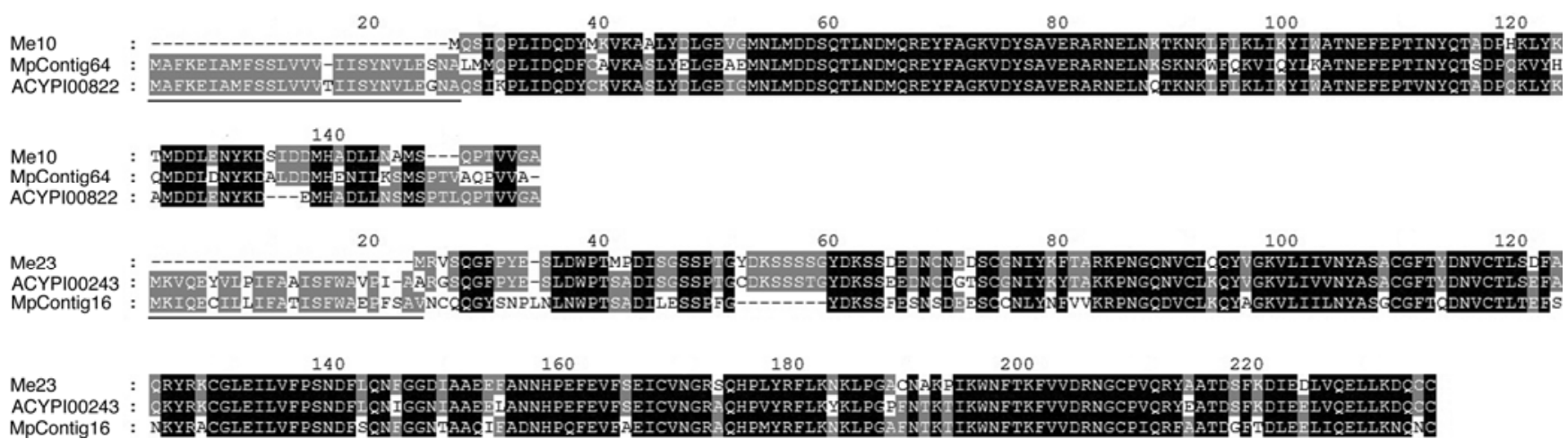

Fig. 5. Alignment of deduced amino acid sequences of Macrosiphum euphorbiae effectors Me10 and Me23, with putative orthologs from Acyrthosiphon pisum (ACYP) and Myzus persicae (Mp). Black and gray shades indicate identical and highly conserved amino acids, respectively. Putative secretion signal peptide sequences are underlined. 
formed into Agrobacterium tumefaciens GV3101 for transient overexpression in $N$. benthamiana. A subset of these sequences were also recombined into the pVSP_PsSPdes vector (Rentel et al. 2008) and were sequenced and transformed into DC3000/ $\Delta a v r P t o / \triangle a v r P t o B$ ( $P$. syringae pv. tomato) for the assay in tomato plants (Nguyen et al. 2010).

\section{Aphid bioassay on $N$. benthamiana.}

Recombinant Agrobacterium tumefaciens containing candidate effector or GFP were grown overnight in Luria Bertani media supplemented with rifampicin $(25 \mu \mathrm{g} / \mathrm{ml})$, gentamycin $(14 \mu \mathrm{g} / \mathrm{ml})$, and kanamycin $(50 \mu \mathrm{g} / \mathrm{ml})$ at $28^{\circ} \mathrm{C}$. The cultures were diluted to a final optical density at $600 \mathrm{~nm}$ of $0.3 \mathrm{in}$ an induction buffer $(10 \mathrm{mM} \mathrm{MES}, 10 \mathrm{mM} \mathrm{MgCl} 2,150 \mu \mathrm{M}$ acetosyringone, $\mathrm{pH}=5.6$ ). Leaves of 4 - to 5-week-old $N$. benthamiana plants were agroinfiltrated using a needleless syringe. Two leaves of four plants were infiltrated per construct. On day 2 after infiltration, four Myzus persicae adults were caged on each infiltrated leaf. The following day, the adults were removed leaving four first-instar nymphs. On day 6, the 4-day-old nymphs were moved to a leaf of a plant infiltrated 2 days earlier. On day 9, the adult aphids were moved again to a leaf of a plant infiltrated 2 days earlier. On day 12, the same adults were moved to a plant infiltrated 2 days earlier. Aphid survival was counted at $6,9,12$, and 15 days after the start of the experiment, and the number of newly produced nymphs was counted on days 9,12 , and 15 . The average number of nymphs produced per adult per day was calculated by dividing the number of nymphs produced by the number of live adult aphids on days 9,12 , and 15 , divided by 2,3 , and 3 , respectively, the number of days between counts.

\section{Aphid bioassay on tomato.}

The $P$. syringae pv. tomato culture was grown on King's B medium $(\mathrm{KBM})$ plates containing rifampicin $(25 \mu \mathrm{g} / \mathrm{ml})$, carbenicillin $(100 \mu \mathrm{g} / \mathrm{ml})$, and kanamycin $(50 \mu \mathrm{g} / \mathrm{ml})$ at $30^{\circ} \mathrm{C}$ for 2 days. A single colony was inoculated into $200 \mu \mathrm{l}$ of liquid KBM, was plated onto KBM, and was incubated for another day at $30^{\circ} \mathrm{C}$. A good healthy lawn on the plate was resuspended in $10 \mathrm{mM} \mathrm{MgCl}$. Whole plants were vacuum infiltrated with $P$. syringae pv. tomato $\left(1 \times 10^{3} \mathrm{CFU} / \mathrm{ml}\right)$ containing a candidate effector or GUS in $1 \mathrm{mM} \mathrm{MgCl}_{2}$ and $0.02 \%$ Silwet L-77. Plants were infested with nine 1-day-old adult M. euphorbiae $24 \mathrm{~h}$ after infiltration. Five plants were used per construct. Aphid fecundity was assessed by counting the number of nymphs and removing them daily for 5 days.

\section{Gene expression analysis.}

Semiquantitative reverse transcription PCR analysis was performed using cDNA prepared from whole aphids or dissected aphid heads and salivary glands or guts using gene-specific primer pairs (Supplementary Table S4). C002 (Mutti et al. 2008) and Sucrase (Price et al. 2007) were used as tissue-specific markers and the ribosomal gene L27 (NM_001126221) was used as internal control. PCR was performed in $25 \mu \mathrm{l}$ with $40 \mathrm{ng}$ of cDNA template, $1 \times$ PCR buffer, $2.5 \mathrm{mM} \mathrm{MgCl}_{2}, 0.4$ mM dNTPs, $2 \mathrm{U}$ of Taq DNA polymerase, and $10 \mu \mathrm{M}$ of each of the forward and reverse primers. The PCR program was initialized at $94^{\circ} \mathrm{C}$ for $5 \mathrm{~min}$, followed by 23 cycles of $94^{\circ} \mathrm{C}$ for $1 \mathrm{~min}, 58^{\circ} \mathrm{C}$ for $45 \mathrm{~s}$, and $72^{\circ} \mathrm{C}$ for $1 \mathrm{~min}$, with a final extension at $72^{\circ} \mathrm{C}$ for $10 \mathrm{~min}$.

\section{Statistical analyses.}

Statistical analysis with aphid data from $N$. benthamiana plants was performed using the one-tailed Flinger-Policello test followed by Bonferrioni adjustment (BenMamoun 2006). Student's $t$-test was used for aphid data from tomato plants.

\section{ACKNOWLEDGMENTS}

We thank A. Collmer (Cornell University, Ithaca, NY, U.S.A.) for $P$. sy-

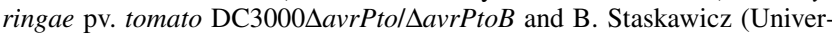
sity of California Berkeley, U.S.A.) for the pVSP_PsSPdes vector. We are also grateful to G. Walker (University of California Riverside) and N. Mutti (DuPont Agricultural Technology, Wilmington, DE, U.S.A.) for helpful discussions. This work was support in part by grants from University of California Experimental Station to I. Kaloshian and United States Department of Agriculture National Institute of Food and Agriculture (award number 2010-65106-20675) to I. Kaloshian and T. Girke.

\section{LITERATURE CITED}

Abramovitch, R. B., Anderson, J. C., and Martin, G. B. 2006. Bacterial elicitation and evasion of plant innate immunity. Nature Rev. Mol. Cell Biol. 7:601-611.

Atamian, H. S., and Kaloshian, I. 2012. Construction of RNA-Seq libraries from large and microscopic tissues for the Illumina sequencing platform. in: RNA Abundance Analysis, H. Jin and W. Gassmann, eds. Humana Press, Totowa. NJ, U.S.A.

Bao, E., Jiang, T., Kaloshian, I., and Girke, T. 2011. SEED: Efficient clustering of next-generation sequences. Bioinformatics 27:2502-2509.

BenMamoun, M. 2006. FPRANK: Stata module to compute Two-Sample Fligner-Policello Robust Rank Order Test. Statistical Software Components, S456739 Department of Economics, Boston College, Chestnut Hill, MA, U.S.A.

Bhattarai, K. K., Xie, Q.-G., Pourshalimi, D., Younglove, T., and Kaloshian, I. 2007. Coil-dependent signaling pathway is not required for $\mathrm{Mi}-1$-mediated potato aphid resistance. Mol. Plant-Microbe Interact. 20:276-282.

Birch, P. R., Rehmany, A. P., Pritchard, L., Kamoun, S., and Beynon, J. L. 2006. Trafficking arms: Oomycete effectors enter host plant cells. Trends Microbiol. 14:8-11.

Blackman, R. L., and Eastop, V. F. 2000. Aphids on the World's Crops. Wiley-Interscience, Chichester, U.K.

Bos, J. I., Prince, D., Pitino, M., Maffei, M. E., Win, J., and Hogenhout, S. A. 2010. A functional genomics approach identifies candidate effectors from the aphid species Myzus persicae (green peach aphid). PLoS Genet. 6:e1001216. Published online.

Buttner, D., and He, S. Y. 2009. Type III protein secretion in plant pathogenic bacteria. Plant Physiol. 150:1656-1664.

Carolan, J. C., Fitzroy, C. I., Ashton, P. D., Douglas, A. E., and Wilkinson, T. L. 2009. The secreted salivary proteome of the pea aphid Acyrthosiphon pisum characterised by mass spectrometry. Proteomics 9:2457-2467.

Carolan, J. C., Caragea, D., Reardon, K. T., Mutti, N. S., Dittmer, N., Pappan, K., Cui, F., Castaneto, M., Poulain, J., Dossat, C., Tagu, D., Reese, J. C., Reeck, G. R., Wilkinson, T. L., and Edwards, O. R. 2011. Predicted effector molecules in the salivary secretome of the pea aphid (Acyrthosiphon pisum): A dual transcriptomic/proteomic approach. J. Proteome Res. 10:1505-1518.

Cerato, C., Rongai, D., Borgatti, S., and Tamba, M. L. 1994. Study of the aphid populations and virus diseases on seed potato crops. Inf. Agrag. 50:67-72.

Chan, C. K., Forbes, A. R., and Raworth, D. A. 1991. Aphid-transmitted viruses and their vectors of the world. Agric. Can. Tech. Bull. 3E:1216.

Cornelis, G. R., and Van Gijsegem, F. 2000. Assembly and function of type III secretory systems. Annu. Rev. Microbiol. 54:735-774.

Davis, E. L., Hussey, R. S., Mitchum, M. G., and Baum, T. J. 2008. Parasitism proteins in nematode-plant interactions. Curr. Opin. Plant Biol. 11:360-366.

De Vos, M., and Jander, G. 2009. Myzus persicae (green peach aphid) salivary components induce defence responses in Arabidopsis thaliana. Plant Cell Environ. 32:1548-1560.

De Vos, M., Van Oosten, V. R., Van Poecke, R. M. P., Van Pelt, J. A., Pozo, M. J., Mueller, M. J., Buchala, A. J., Metraux, J.-P., Van Loon, L. C., Dicke, M., and Pieterse, C. M. J. 2005. Signal signature and transcriptome changes of Arabidopsis during pathogen and insect attack. Mol. Plant-Microbe Interact. 18:923-937.

Earley, K. W., Haag, J. R., Pontes, O., Opper, K., Juehne, T., Song, K., and Pikaard, C. S. 2006. Gateway-compatible vectors for plant functional genomics and proteomics. Plant J. 45:616-629.

Emanuelsson, O., Nielsen, H., Brunak, S., and von H eijne, G. 2000. Predicting subcellular localization of proteins based on their N-termina amino acid sequence. J. Mol. Biol. 300:1005-1016.

Giordanengo, P., Brunissen, L., Rusterucci, C., Vincent, C., van Bel, A., Dinant, S., Girousse, C., Faucher, M., Bonnemain, J. L. 2010. Compatible plant-aphid interactions: How do aphids manipulate plant responses. C. R. Biol. 333:516-523. 
Goodin, M. M., Zaitlin, D., Naidu, R. A., and Lommel, S. A. 2008. Nicotiana benthamiana: Its history and future as a model for plant-pathogen interactions. Mol. Plant-Microbe Interact. 21:1015-1026.

Gosset, V., Harmel, N., Gobel, C., Francis, F., Haubruge, E., Wathelet, J. P., du Jardin, P., Feussner, I., and Fauconnier, M. L. 2009. Attacks by a piercing-sucking insect (Myzus persicae Sultzer) or a chewing insect (Leptinotarsa decemlineata Say) on potato plants (Solanum tuberosum L.) induce differential changes in volatile compound release and oxylipin synthesis. J. Exp. Bot. 60:1231-1240.

Guttman, D. S., and Greenberg, J. T. 2001. Functional analysis of the Type III effectors AvrRpt2 and AvrRpm1 of Pseudomonas syringae with the use of a single-copy genomic integration system. Mol. Plant-Microbe Interact. 14:145-155.

Harmel, N., Letocart, E., Cherqui, A., Giordanengo, P., Mazzucchelli, G., Guillonneau, F., De Pauw, E., Haubruge, E., and Francis, F. 2008. Identification of aphid salivary proteins: A proteomic investigation of Myzus persicae. Insect Mol. Biol. 17:165-174

Harris, M. O., Stuart, J. J., Mohan, M., Nair, S., Lamb, R. J., and Rohfritsch, O. 2003. Grasses and gall midges: Plant defense and insect adaptation. Annu. Rev. Entomol. 48:549-577.

Hogenhout, S. A., and Bos, J. I. 2011. Effector proteins that modulate plant-insect interactions. Curr. Opin. Plant Biol. 14:422-428.

Horne, I., Haritos, V. S., and Oakeshott, J. G. 2009. Comparative and functional genomics of lipases in holometabolous insects. Insect Biochem. Mol. Biol. 39:547-567.

International Aphid Genomics Consortium. 2010. Genome sequence of the pea aphid Acyrthosiphon pisum. PLoS Biol. 8:e1000313. Published online.

Jones, J. D., and Dangl, J. L. 2006. The plant immune system. Nature 444:323-329.

Kaloshian, I., and Walling, L. L. 2005. Hemipterans as plant pathogens Annu. Rev. Phytopathol. 43:491-521.

Kamoun, S. 2006. A catalogue of the effector secretome of plant pathogenic oomycetes. Annu. Rev. Phytopathol. 44:41-60.

Kempema, L. A., Cui, X., Holzer, F. M., and Walling, L. L. 2007. Arabidopsis transcriptome changes in response to phloem-feeding silverleaf whitefly nymphs. Similarities and distinctions in responses to aphids. Plant Physiol. 143:849-865.

Kim, J. H., and Jander, G. 2007. Myzus persicae (green peach aphid) feeding on Arabidopsis induces the formation of a deterrent indole glucosinolate. Plant J. 49:1008-1019.

Lamb, C., and Dixon, R. A. 1997. The oxidative burst in plant disease resistance. Annu. Rev. Plant Physiol. Plant Mol. Biol. 48:251-275.

Mantelin, S., Peng, H. C., Li, B., Atamian, H. S., Takken, F. L., and Kaloshian, I. 2011. The receptor-like kinase SISERK1 is required for $\mathrm{Mi}$ 1-mediated resistance to potato aphids in tomato. Plant J. 67:459-471.

Margaritopoulos, J. T., Kasprowicz, L., Malloch, G. L., and Fenton, B. 2009. Tracking the global dispersal of a cosmopolitan insect pest, the peach potato aphid. BMC Ecol. 9:13.

Martinez de Ilarduya, O., Xie, Q., and Kaloshian, I. 2003. Aphid-induced defense responses in $\mathrm{Mi}-1$-mediated compatible and incompatible tomato interactions. Mol. Plant-Microbe Interact. 16:699-708.

Miles, P. 1999. Aphid saliva. Biol. Rev. 74:41-85.

Moeller, F. W. 1973. The host plants of the potato aphid Macrosiphum euphorbiae and of closely related species. Wiss. Z. Univ. Rostock Math. Naturwiss. Reihe 22:1179-1184.

Moran, N. A. 1992. The evolution of aphid life cycles. Annu. Rev. Entomol. 37:321-348

Moran, P. J., Cheng, Y., Cassell, J. L., and Thompson, G. A. 2002. Gene expression profiling of Arabidopsis thaliana in compatible plant-aphid interactions. Arch. Insect Biochem. Physiol. 51:182-203.

Mutti, N. S., Louis, J., Pappan, L. K., Pappan, K., Begum, K., Chen, M. S. Park, Y., Dittmer, N., Marshall, J., Reese, J. C., and Reeck, G. R. 2008. A protein from the salivary glands of the pea aphid, Acyrthosiphon pisum, is essential in feeding on a host plant. Proc. Natl. Acad. Sci. U.S.A. 105:9965-9969.

Nguyen, H. P., Chakravarthy, S., Velasquez, A. C., McLane, H. L., Zeng, L., Nakayashiki, H., Park, D.-H., Collmer, A., and Martin, G. B. 2010. Methods to study PAMP-triggered immunity using tomato and Nicotiana benthamiana. Mol. Plant-Microbe Interact. 23:991-999.

Petersen, T. N., Brunak, S., von Heijne, G., and Nielsen, H. 2011. SignalP 4.0: Discriminating signal peptides from transmembrane regions. Nat. Methods 8:785-786

Pollard, D. G. 1973. Plant penetration by feeding aphids (Hemiptera, Aphidoidea): A review. Bull. Entomol. Res. 62:631-714.

Ponsen, M. B. 1972. The site of potato leafroll virus multiplication in its vector, Myzus persicae: An anatomical study. Mededelingen Landbouw- hogeschool Wageningen 72:1-147.

Price, D. R., Karley, A. J., Ashford, D. A., Isaacs, H. V., Pownall, M. E., Wilkinson, H. S., Gatehouse, J. A., and Douglas, A. E. 2007. Molecular characterisation of a candidate gut sucrase in the pea aphid, Acyrthosiphon pisum. Insect Biochem. Mol. Biol. 37:307-317.

Radcliffe, E. B., and Ragsdale, D. W. 2002. Aphid-transmitted Potato viruses: The Importance of Understanding Vector Biology. Am. J. Potato Res. 79:353-386.

Rentel, M. C., Leonelli, L., Dahlbeck, D., Zhao, B., and Staskawicz, B. J. 2008. Recognition of the Hyaloperonospora parasitica effector ATR13 triggers resistance against oomycete, bacterial, and viral pathogens. Proc. Natl. Acad. Sci. U.S.A. 105:1091-1096.

Rodriguez-Saona, C. R., Musser, R. O., Vogel, H., Hum-Musser, S. M., and Thaler, J. S. 2010. Molecular, biochemical, and organismal analyses of tomato plants simultaneously attacked by herbivores from two feeding guilds. J. Chem. Ecol. 36:1043-1057.

Rossi, M., Goggin, F. L., Milligan, S. B., Kaloshian, I., Ullman, D. E., and Williamson, V. M. 1998. The nematode resistance gene $M i$ of tomato confers resistance against the potato aphid. Proc. Natl. Acad. Sci. U.S.A. 95:9750-9754.

Schulz, M. H., Zerbino, D. R., Vingron, M., and Birney, E. 2012. Oases: Robust de novo RNA-seq assembly across the dynamic range of expression levels. Bioinformatics 28:1086-1092.

Segonzac, C., and Zipfel, C. 2011. Activation of plant pattern-recognition receptors by bacteria. Curr. Opin. Microbiol. 14:54-61.

Singh, R. P., and Boiteau, G. 1986. Reevaluation of the potato aphid, Macrosiphum euphorbiae (Thomas), as vector of potato virus Y. Am. Potato J. 63:335-340.

Singh, V., Louis, J., Ayre, B. G., Reese, J. C., Pegadaraju, V., and Shah, J. 2011. TREHALOSE PHOSPHATE SYNTHASE11-dependent trehalose metabolism promotes Arabidopsis thaliana defense against the phloemfeeding insect Myzus persicae. Plant J. 67:94-104.

Sohn, K. H., Lei, R., Nemri, A., and Jones, J. D. 2007. The downy mildew effector proteins ATR1 and ATR13 promote disease susceptibility in Arabidopsis thaliana. Plant Cell 19:4077-4090.

Thompson, G. A., and Goggin, F. L. 2006. Transcriptomics and functional genomics of plant defence induction by phloem-feeding insects. J. Exp. Bot. 57:755-766

Tjallingii, W. F. 2006. Salivary secretions by aphids interacting with proteins of phloem wound responses. J. Exp. Bot. 57:739-745.

Tjallingii, W. F., and Hogen Esch, T. 1993. Fine structure of aphid stylet routes in plant tissues in correlation with EPG signals. Physiol. Entomol. 18:317-328.

van der Hoorn, R. A. 2008. Plant proteases: From phenotypes to molecular mechanisms. Annu. Rev. Plant Biol. 59:191-223.

Van Emden, H. F., and Harrington, R. 2007. Aphids As Crop Pests. Eds. CABI, Wallingford, U.K

Von Dohlen, C. D., Rowe, C. A., and Heie, O. E. 2006. A test of morphological hypotheses for tribal and subtribal relationships of Aphidinae (Insecta: Hemiptera: Aphididae) using DNA sequences. Mol. Phylogenet. Evol. 38:316-329.

Weidemann, H. L. 1968. Zur morohologie der hauptspeicheldruse von Myzus persicae (Sulz.). Entomol. Exp. Appl. 11:450-454.

Will, T., Tjallingii, W. F., Thonnessen, A., and van Bel, A. J. 2007. Molecular sabotage of plant defense by aphid saliva. Proc. Natl. Acad. Sci U.S.A. 104:10536-10541.

Wilson, A. C., Sternberg, L. S. L., and Hurley, K. B. 2011. Aphids alter host-plant nitrogen isotope fractionation. Proc. Natl. Acad. Sci. U.S.A. 108:10220-10224.

Zarate, S. I., Kempema, L. A., and Walling, L. L. 2007. Silverleaf whitefly induces salicylic acid defenses and suppresses effectual jasmonic acid defenses. Plant Physiol. 143:866-875.

Zhang, P., Zhu, X., Huang, F., Liu, Y., Zhang, J., Lu, Y., and Ruan, Y. 2011. Suppression of jasmonic acid-dependent defense in cotton plant by the mealybug Phenacoccus solenopsis. PLoS One 6:e22378. Published online.

\section{AUTHOR-RECOMMENDED INTERNET RESOURCES}

The Acyrthosiphon pisum EST database: www.aphidests.org

The Aphid genome database: www.aphidbase.com/aphidbase

Center for Biological Sequence Analysis TargetP prediction server: www.cbs.dtu.dk/services/TargetP

NCBI Conserved Domain database:

www.ncbi.nlm.nih.gov/Structure/cdd/wrpsb.cgi

SignalP4.0 server: www.cbs.dtu.dk/services/SignalP 